\title{
Pure elderly suicide rates versus combined pure elderly suicide, accidental and undetermined death rates: methodological issues in cross-national studies
}

The verdict of suicide depends upon local legal definitions, culture, and reporting practices and procedures (Shah and Ganesvaran, 1994). Legal definitions of suicide vary across countries. In Austria, officers of the Federal Statistics Division may count a death as suicide if it is suspected as such, even without legal evidence. In Luxembourg, a suicide note is required for a verdict of suicide. In the U.K. and Australia, the verdict of suicide is reached by the coroner, who must be satisfied that suicide has been proved beyond reasonable doubt; if there is reasonable doubt, then a verdict of undetermined death or an open verdict is returned. Owing to the possibility of some probable suicides being misclassified as accidental and undetermined deaths (Chishti et al., 2003), it has been suggested that accidental and undetermined deaths should also be utilized in suicide research (O'Donnell and Farmer, 1995). However, there is evidence from European Union countries that misclassification contributes to only a minor proportion of the variation in general population suicide rates (Chishti et al., 2003), although this has not been specifically examined for elderly suicide rates.

A study to examine the hypothesis that misclassification of elderly suicides may explain cross-national variations in elderly suicide rates was undertaken using two approaches. First, if some suicides were misclassified as undetermined deaths then there would be an inverse correlation between elderly suicide rates and rates of accidental deaths and undetermined deaths in the elderly. Second, the relationship between variables, previously reported to be associated with pure elderly suicide rates in a cross-national study (Shah et al., 2007), and the combined rates for elderly suicides and accidental deaths and undetermined deaths may be different to that observed for pure elderly suicide rates if suicides were misclassified as accidental deaths and undertermined deaths.

Data on pure suicide rates and rates of accidental deaths and undertermined deaths for males and females in the age-bands 65-74 years and 75+ years were ascertained from the World Health Organization (WHO) website (www.who. int/whois/database/mort/table1.cfm) for the latest available year (median 2000, range 1985-2003). The WHO website (www.who.int/whois/database/mort/ table1.cfm) also provided data on the elderly ( $65+$ years) population size and the total population size, and this allowed calculation of the proportion of elderly in the total population. The WHO website (www.who.int/countries/en/) provided data on the male and female life expectancy, per capita gross national domestic product (GDP), the proportion of GDP spent on health, per capita expenditure on health, and male and female child mortality rates (i.e. mortality before the age of five years) for the year 2002. The United Nations Development Programme website (www.hdr.undp.org/reports/global/2005/pdf/hdr05_HDI.pdf) provided data on a measure of income inequality called the Gini coefficient. 
The relationship between pure elderly suicide rates and rates of accidental deaths and undertermined deaths in the elderly across different countries was examined using Spearman's correlation coefficient. The main findings from an earlier study of correlates of pure elderly suicide rates, using the same data set (Shah et al., 2007), were: (i) a significant positive correlation between the elderly population size and the proportion of elderly in the population and suicide rates for both sexes in both of the elderly age-bands; (ii) a significant negative correlation between the Gini coefficient and suicide rates for both sexes in both of the elderly age-bands; (iii) a significant positive correlation between per capita expenditure on health and suicide rates for both sexes in both of the elderly age-bands; (iv) a significant positive correlation between suicide rates and the proportion of GDP spent on health was evident in males in both of the elderly age-bands; (v) a significant negative correlation between child mortality rates and suicide rates for both sexes in both of the elderly age-bands; and (vi) a significant positive correlation between life expectancy and suicide rates was evident in females in both of the elderly age-bands. The relationship between these variables and the combined rates of elderly suicides, accidental deaths and undertermined deaths was examined using Spearman's correlation coefficient.

Complete data sets were available for 49 countries. There was no significant correlation between pure suicide rates and rates of accidental deaths and undetermined deaths in males aged 65-74 years. There were positive correlations between pure suicide rates and rates of accidental deaths and undetermined deaths in males aged 75+ years $(\rho=+0.5, \mathrm{p}<0.00001)$, females aged 65-74 years $(\rho=+0.47, \mathrm{p}=0.001)$ and females aged $75+$ years $(\rho=+0.48, \mathrm{p}=0.001)$.

Table S1 (published online as supplementary material attached to this letter at www.journals.cambridge.org/jid_IPG) illustrates the relationship between combined rates for pure suicides, accidental deaths and undertermined deaths in both sexes in both the elderly age-bands. Most of the findings were significantly different from those previously reported for pure suicide rates (Shah et al., 2007).

These findings do not support previous reports that the variance in cross-national suicide rates is influenced by rates of accidental deaths and undetermined deaths. Moreover, the correlates of pure suicide rates and of combined rates for pure suicides, accidental deaths and undertermined deaths were significantly different. This is important in interpreting findings from different cross-national studies using different methods of calculating suicide rates. The latter finding also highlights how the results can change by using different methods of calculating suicide rates. These findings therefore question the appropriateness of using either method of calculating suicide rates in crossnational studies with an ecological design.

\section{References}

Chishti, P., Stone, D. H., Corcoran, P., Willianson, E., Petridou, E., EUROSAVE Working Group (2003). Suicide mortality in the European Union. European fournal of Public Health, 13, 108-114.

O'Donnell, I. and Farmer, R. (1995). The limitations of official suicide statistics. British Fournal of Psychiatry, 166, 458-461. 
Shah, A. K. and Ganesvaran, T. (1994). Suicide in the elderly. In E. Chiu and D. Ames (eds.), Functional Psychiatric Disorders of the Elderly (pp. 221-244), Cambridge: Cambridge University Press.

Shah, A. K., Bhatt, R., MacKenzie, S. and Koen, C. (2007). A cross-national study of the relationship between elderly suicide rates and life expectancy and markers of socioeconomic status and healthcare. International Psychogeriatrics. DOI: 10.1017/S1041610207005352 (e-published ahead of print).

\section{AJIT SHAH}

Professor of Ageing, Ethnicity and Mental Health, University of Central Lancashire, U.K. and Consultant Psychiatrist, West London Mental Health NHS Trust, London, U.K.

Email: ajit.shah@wlmht.nhs.uk

\begin{tabular}{|c|c|c|c|c|}
\hline & $\begin{array}{l}\text { MALES } \\
65-74 \\
\text { YEARS }\end{array}$ & $\begin{array}{l}\text { MALES } \\
75+ \\
\text { YEARS }\end{array}$ & $\begin{array}{l}\text { FEMALES } \\
65-74 \\
\text { YEARS }\end{array}$ & $\begin{array}{l}\text { FEMALES } \\
75+ \\
\text { YEARS }\end{array}$ \\
\hline GDP & $\begin{array}{l}\rho=-0.35 \\
\mathrm{P}=0.014\end{array}$ & NS & NS & $\begin{array}{l}\rho=+0.39 \\
\mathrm{P}=0.006\end{array}$ \\
\hline Gini coefficient & NS & $\begin{array}{l}\rho=-0.37 \\
\mathrm{P}=0.013\end{array}$ & $\begin{array}{l}\rho=-0.41 \\
\mathrm{P}=0.006\end{array}$ & $\begin{array}{l}\rho=-0.52 \\
\mathrm{P}<0.00001\end{array}$ \\
\hline Proportion of GDP spent on health & $\begin{array}{l}\rho=-0.37 \\
\mathrm{P}=0.013\end{array}$ & NS & NS & $\begin{array}{l}\rho=+0.28 \\
\mathrm{P}=0.051\end{array}$ \\
\hline Per capita health expenditure & $\begin{array}{l}\rho=-0.4 \\
\mathrm{P}=0.005\end{array}$ & NS & NS & $\begin{array}{l}\rho=+0.43 \\
\mathrm{P}=0.002\end{array}$ \\
\hline Male life expectancy & $\begin{array}{l}\rho=-051 \\
\mathrm{P}<0.0001\end{array}$ & NS & & \\
\hline Female life expectancy & & & NS & $\begin{array}{l}\rho=+0.44 \\
\mathrm{P}=0.02\end{array}$ \\
\hline Male child mortality rates & NS & $\begin{array}{l}\rho=-0.42 \\
\mathrm{P}=0.003\end{array}$ & & \\
\hline Female child mortality rates & & & NS & $\begin{array}{l}\rho=-0.57 \\
\mathrm{P}<0.00001\end{array}$ \\
\hline Total elderly population & $\begin{array}{l}\rho=-0.34 \\
\mathrm{P}=0.017\end{array}$ & NS & NS & NS \\
\hline $\begin{array}{l}\text { Proportion of elderly in the general } \\
\text { population }\end{array}$ & NS & $\begin{array}{l}\rho=+0.35 \\
P=0.015\end{array}$ & NS & $\begin{array}{l}\rho=+0.39 \\
P=0.006\end{array}$ \\
\hline
\end{tabular}

NS $=$ Not significant 